\title{
ENGEVISTA
}

Página da revista: http://www.uff.br/engevista/seer/

\section{Análise dos indicadores de desempenho de um centro de distribuição de bebidas}

\section{Analysis of performance indicators of a beverage distribution center}

\author{
Jéssica Almeida Abijaude ${ }^{1}$ \\ Aline Patrícia Mano ${ }^{2}$ \\ Wesley Vieira Silva ${ }^{3}$ \\ Diego de Castro Fettermann ${ }^{4}$
}

Resumo: As empresas têm utilizado cada vez mais os indicadores de desempenho como meio de monitorar seus resultados. Conhecer o comportamento dos processos é importante para busca dos resultados operacionais almejados. Sendo assim, este trabalho apresenta um estudo sobre o comportamento de três indicadores de desempenho operacionais utilizados em um Centro de Distribuição Direta e sua contribuição para o desempenho operacional. Os indicadores selecionados foram: percentual de vendas efetuadas aos clientes da base, percentual de devolução e percentual de fora de rota. Partindo da hipótese de que esses indicadores refletem o volume de vendas, com o auxilio do software livre GrETL, foi analisado o modelo dos mínimos quadrados, depois realizado o teste de RESET, a análise de correlação e por fim o ajuste utilizando Cochrane- Orcutt. De acordo com os dados analisados aceitou-se a hipótese inicial, revelando que o modelo composto pelas 3 variáveis explica $51 \%$ do volume de vendas.

Palavras-chave: indicadores de desempenho, séries temporais, testes estatísticos.

\footnotetext{
${ }^{1}$ UESC - Universidade Estadual de Santa Cruz

${ }^{2}$ UESC - Universidade Estadual de Santa Cruz/PUCPR - Pontificia Universidade Catolica do Paraná

${ }^{3}$ PUCPR - Pontificia Universidade Catolica do Paraná

${ }^{4}$ UFSC - Universidade Federal de Santa Catarina
}

ENGEVISTA, V. 18, n. 1, p. 125-141, Julho 2016. 
ISSN: $1415-7314$

ISSN online: $2317-6717$

Abstract: Companies have been increasing the use performance indicators as a means to monitor their results. To understand the behavior of processes is important when searching for the desired operating results. Thus, this work presents a study of the behavior of three operational performance indicators used in a Center of Direct Distribution and their contribution to operational performance. The indicators selected were: percentage of sales made to its customer base, percentage of return and percentage of off-route. This is assuming that these indicators reflect the volume of sales, and with the aid of the free software, Gretl, it was considered the model of least squares. Then the RESET test was performed, followed by the correlation analysis and the adjustment was made using Cochrane- Orcut. In accordance with the analyzed data the initial hypothesis was accepted, showing that the model consists of 3 variables explaining $56 \%$ of the sales volume.

Keywords: performance indicators, correlation, direct distribution center. 


\section{Introdução}

O setor de prestação de serviços apresenta relevante participação na economia de um país, e é marcado pela competitividade entre as empresas que nele atuam (Silva et al, 2012). As organizações buscam constantemente um grau de excelência em serviços, considerando os clientes como peça fundamental para a conquista e manutenção dos mercados (Freitas, 2005). Frente a este cenário, as empresas do ramo de serviços passaram a adotar programas, ferramentas e métodos da qualidade como meio de atingir a maior eficiência e lucratividade, e fomentar a contínua melhoria de seus processos. Aliado a preocupação com a satisfação do cliente, medir constantemente o desempenho de suas ações se torna essencial, para assegurar resultados consistentes e eficazes.

No entanto, mesmo que o engenheiro de produção tenha habilidades para trabalhar tanto nos setores manufatureiros quanto nos setores de serviços (Masculo, 2006), os estudos em engenharia são geralmente voltados para soluções de problemas apresentados pela indústria, o que leva algumas empresas do ramo de serviços a adaptarem técnicas utilizadas nas indústrias para implantação em seus processos, fato este que, muitas vezes, tem gerado resultados positivos (Francischini et al, 2006). O crescimento do setor de serviços provocou a necessidade de uma gestão de seus sistemas internos, voltados para o aumento da qualidade em todo o cenário organizacional, com a aplicação de métodos e ferramentas que tenham um impacto positivo em seu desempenho operacional.

Independente da natureza da empresa, seja de prestação de serviço ou produção de bens, medir o desempenho de seus processos e/ou produtos/serviços se torna essencial para compreensão dos seus resultados servindo também como base para a melhoria contínua. Porém, para cada tipo de organização existe uma maneira particular de abordar o tema, uma vez que a dinâmica e os objetivos organizacionais são diferentes. No caso da prestação de serviços, manter a qualidade da ação fornecida à parte interessada torna-se um desafio pela intangibilidade existente e pelo fato do serviço ser produzido e consumido simultaneamente (Souza, 2006).

Um exemplo de estruturas prestadoras de serviços são os Centros de Distribuição Direta (CDD), que são adotados por empresas como unidade de negócio localizada estrategicamente para distribuir os seus produtos com maior facilidade e agilidade (Santos, 2006). Além disso, há CDD's que incorporam em sua estrutura o processo de vendas, responsável por manter um contato direto com os clientes e aproximá-los do negócio da empresa, construindo um relacionamento mais estreito e consistente. Nesse contexto, os CDD's enfrentam grande desafio em seus processos e o monitoramento de seu desempenho deve focar na garantia da qualidade do serviço prestado, além de eficiência.

De acordo com Gonçalves (2002), ao utilizar indicadores para a medição de desempenho de uma empresa, há a possibilidade de se obter um maior conhecimento sobre seus processos, relacionados com os pontos críticos de sucesso. Para tal, é de extrema importância que o desempenho dos mesmos reflitam o resultado obtido. 
Kaplan e Norton (2004) afirmam que para haver uma gestão eficiente dos processos, é fundamental que os indicadores possuam conexão e uma relação de causalidade entre ações e resultados. Assim, é indispensável para a estratégia da empresa que suas perspectivas, metas e indicadores possuam inter-relação para que os ativos intangíveis se transformem em resultados satisfatórios tanto para os clientes quanto para a empresa.

O objetivo deste trabalho é analisar três principais indicadores de desempenho de um Centro de Distribuição Direta, que dizem respeito à área de vendas e logística, e avaliar se eles refletem o resultado do volume de vendas. Para tal, auxiliado pelo software GRETL, se utilizou o modelo dos mínimos quadrados, o teste de RESET, análise de correlação e o modelo de Cochrane-Orcutt.

\section{Revisão bibliográfica}

Considerando que sejam temas pertinentes a serem abordados e que agreguem de fato valor teórico para esta pesquisa, foi realizado um levantamento de definições e informações a cerca de: Centro de Distribuição Direta (CDD), medição de desempenho, indicadores de desempenho (KPI), Balanced Score Card e correlação.

\subsection{Centros de distribuição (CD)}

Diferentes autores (Wanke et al, 2006; Silva, 2006; Santos, 2006) concordam que os Centros de Distribuição surgiram como estratégia para empresas que buscam vantagens geográficas, redução no tempo de entrega dos produtos e baixo custo. O numero de empresa que adotam os CD's como unidade de negócio cresce consideravelmente.

Os centros de distribuição são, de certa forma, uma evolução dos depósitos, pois a ênfase é dada não apenas no armazenamento dos produtos, mas em seu fluxo (desde a chegada ao armazém, até a saída) e em atividades de administração internas, com foco no ganho de produtividade e baixo custo operacional (Côrtez, 2006). Além de armazenar os produtos, o CD é responsável por garantir que estes estejam em constante movimento da melhor maneira possível.

Para o gerenciamento do fluxo de materiais e informações e consolidação dos requerimentos dos clientes para posterior distribuição física há empresas que adotam a estrutura de Centro de Distribuição Direta (CDD), que exige um maior controle de tempo no processamento do pedido e na entrega, e controle das avarias que o produto possa vir a ter (Wanke et al, 2006). A movimentação dos produtos acontecem a partir da geração dos pedidos realizados pelo processo de vendas direta, que deve garantir que o planejamento de produção realizado e a previsão de demanda estejam alinhados, evitando grande formação de estoque ou falta de produtos.

As vendas em um centro de distribuição servem como forma de aproximar os clientes da empresa. No caso de centros de distribuição de bebidas é possível atender diferentemente os clientes de acordo ao seu tipo de atuação no mercado e potencial de revenda dos produtos ao consumidor final. Isso influenciará diretamente no seu preço praticado e quantidade mínima exigida de compra e 
venda em um determinado período. A presença de um vendedor no mercado garante que estes tipos de especificações sejam atendidas e que a geração do pedido aconteça a partir de negociação, em que o vendedor tem a oportunidade de garantir uma maior participação da empresa no mercado (marketing share) e a satisfação do cliente em relação a prestação de serviço, fidelizando-o.

Para garantir a realização adequada dos procedimentos e obter resultados eficientes em um Centro de Distribuição Direta, é possível adotar ferramentas de medição de desempenho.

\subsection{Mediação de desempenho}

Segundo Tezza, (2010), até o inicio dos anos de 1980, o desempenho das empresas era medido a partir de seu status financeiro. Ainda segundo a autora, uma das primeiras abordagens não financeira e organizacional foi a do 7-S da McKinsey, que considera sete elementos organizacionais e os subdivide em objetivos (estratégia, estrutura e sistema) e subjetivos (habilidades, valor compartilhado, estilo e pessoas). Este tipo de abordagem promove uma visão mais abrangente da organização, o que possibilita ampliar os horizontes de possibilidades e oportunidades.

Em geral, os estudos acerca de métodos de medição de desempenho sempre foram mais voltados para os setores manufatureiros. Segundo Tezza (2010), as abordagens puramente em serviços iniciaram-se primeiramente voltadas para a qualidade sob o ponto de vista do cliente. Um exemplo desse tipo de abordagem é o modelo SERVQUAL proposto por Parasuraman et al. (1988), no qual sugere-se um conjunto de 22 atributos com objetivo de auxiliar os prestadores de serviço a entenderem melhor as expectativas do mercado e necessidade dos clientes, e, consequentemente, poderem melhorar seu nível de serviço.

Independente do setor ao qual a organização está inserida, a necessidade de desenvolver um pensamento criativo e inovador e se manter constantemente atualizada são constantes. Os processos necessitam de controle e monitoramento para garantir a qualidade do produto/serviço exigida pelos clientes. Para o monitoramento do desempenho desses processos, são desenvolvidos indicadores de desempenho considerando os principais fatores que compõem a organização e que serão termômetro para o reconhecimento do sucesso ou fracasso da organização. (Freitas, 2011)

O desenvolvimento de um sistema de medição com ênfase na integração das medições nos diferentes níveis da organização, de forma consistente com os objetivos estratégicos, pode contribuir de maneira decisiva para a efetiva implementação e consolidação de estratégias de melhoria do desempenho organizacional, impulsionando as necessárias mudanças, inclusive a crítica mudança da cultura organizacional. (Soares et al, 1999). Há diversos modelos para a medição de desempenho na literatura, os quais o Balanced Score Card é um dos mais conhecidos e citados (Kaplan \& Norton, 2007). 


\subsection{Balanced Scorecard (BSC)}

O Balanced Scorecard (BSC) foi proposto inicialmente por Robert Kaplan e David Norton em 1992, e hoje é utilizado por empresas que buscam comunicar e alinhar sua estratégia por todos os seus níveis organizacionais. (Kaplan e Norton, 2007)

O BSC é apresentado como um modelo que integra de maneira balanceada as dimensões críticas para a gestão estratégica da organização, tendo ao centro sua visão e estratégia. O modelo trabalha com indicadores de desempenho associados às metas referentes a todas as dimensões do negócio, levando tal informação a todos os níveis da organização (Soares et al, 1999).

$\mathrm{O}$ método acrescenta às medidas financeiras tradicionais, valores fundamentais para o desempenho organizacional em três principais áreas não financeiras: a relação da empresa com seus clientes, seus processos internos de maior importância, e seu aprendizado e crescimento (Kaplan \& Norton, 2007)

Para Kaplan e Norton (2007), a vantagem da aplicação do BSC em uma organização não é apenas apresentar uma perspectiva mais ampla sobre o bom funcionamento das atividades da empresa, é também um caminho para se obter uma estrutura de organização com grande potencial para competir no mercado.

Embora as medidas apresentadas pelo BSC sejam aplicadas em muitas organizações, não há solução padrão para medir a performance de uma empresa. O tipo de sistema de gestão de desempenho adotado por esta pode variar de acordo com os fatores internos e externos que originaram a necessidade e decisão de implementar tal metodologia, como a posição da empresa no mercado, tipo de negócio e filosofia da organização. (Roest, 1997)

O Balanced Scorecard é um método que uma organização pode se basear para desenvolver e implementar um sistema de gestão de desempenho próprio, levando em consideração seus objetivos específicos e suas particularidades. Para gerir o desempenho operacional de uma empresa, deve-se considerar a definição e utilização de indicadores de desempenho que permite a organização acompanhar seus resultados e reagir as suas variações em tempo hábil.

\subsection{Indicadores de desempenho}

Segundo Soares (1999), mundialmente tem se dado grande importância à questão da medição do desempenho, utilizando grupos de ferramentas chamadas indicadores. Os indicadores podem ser definidos como funções que permitem obter informações sobre as medidas relacionadas a um produto, um processo, um sistema ou uma grandeza ao longo do tempo.

Os indicadores são propostos para medir o desempenho de uma organização em áreas-chave do negócio como clientes, produtos e processos, e devem ser encarados como um meio para auxiliar a gestão da qualidade no controle e identificação de necessidades da empresa. (Martins e Costa Neto, 1998). Identificar esses indicadores não é fácil para a maioria das empresas, e se mal definidos, 
podem conduzir as decisões estratégicas de investimento de recursos a direções equivocadas, causando impacto negativo no seu resultado. (Freitas, 2011)

Segundo Parmenter (2010), há 7 características dos indicadores de desempenho: (i) as medições não são exclusivamente financeiras; (ii) devem possuir frequência de medição, seja ela diária, semanal, mensal, etc. (iv) são utilizados pelo executivo chefe e pela equipe de gestão sênior (v) indica claramente a ação requerida para aquele problema identificado; (vi) são direcionadas para determinadas áreas dentro da empresa, tendo responsável direto identificado facilmente; (vii) tem um impacto significante para a tomada de decisão; (viii) encorajam ações apropriadas.

Segundo Slack et al., (1997) não é possível reduzir a complexidade do desempenho de um negócio a um único indicador. Assim, é necessária a aplicação de diversos indicadores para atingir as diferentes visões dos processos e atividades que compõem uma organização e sua estratégia.

Os indicadores de desempenho representam uma forma de quantificar uma atividade ou processo que a empresa queira medir, seja satisfação do cliente, nível de serviço, velocidade de determinado processo, etc. $\mathrm{O}$ acompanhamento dos indicadores deve ser feito regularmente uma vez que são desenvolvidos para gerir os resultados operacionais. Além disso, os indicadores desenvolvidos devem interagir, de forma que um indicador não influencia negativamente o resultado de um outro indicador. Para conhecer essa interação entre os indicadores, é possível utilizar as analises de correlação.

\section{Método}

Este trabalho apresenta um estudo de caso em um Centro de Distribuição Direta (CDD) de bebidas, com o objetivo de avaliar se os indicadores operacionais que estão sendo utilizados realmente refletem o volume de vendas utilizando para isso análise de séries temporais. .

Para se chegar ao objetivo proposto, o trabalho iniciou-se com uma revisão bibliográfica acerca de definições, conceitos e informações de temas que agregassem valor teórico e influenciassem no desenvolvimento e entendimento, que foram: Centro de Distribuição, Medição de Desempenho, Balanced Scorecard, indicadores de desempenho. Para isto recorreu-se a várias fontes de dados como teses, dissertações, base de dados, periódicos, livros, artigos de congresso, artigos de revistas, etc.

Em seguida, a fase de coleta de dados se deu a partir de observação direta, com anotações periódicas acerca do contexto organizacional. Ou seja, buscou-se obter informações que descrevessem o processo de medição de desempenho da empresa analisada e a possível influência existente entre diferentes indicadores.

Ao fim do período de observação, a análise foi realizada utilizando software livre GRETL ${ }^{\circledR}$.

Os dados utilizados para análise referem-se a indicadores utilizados pela empresa estudada, com periodicidade mensal, para esse trabalho foram utilizados 30 meses de coleta entre os anos de 2013 e 2015. 
Para analisar a série temporal primeiramente foi estimado um modelo usando a técnica dos mínimos quadrados, e por conseguinte foi utilizado o mesmo modelo com a exclusão da constante, por meio desses resultados analisou -se a hipótese $\mathrm{H}_{0}$ que afirmava que nenhuma das variáveis independentes afetavam o volume de vendas.

Em seguida foi utilizado o teste de RESET ( Regression Equation Specification Error Test) cujo objetivo foi verificar se havia erros de especificação no modelo, nesse caso $\mathrm{H}_{0}$ afirmava que a especificação do modelo estava adequada.

Após a realização do teste de RESET foi feito o teste de White a fim de verificar a homocedasticidade.

O passo seguinte consistiu na análise da correlação entre as variáveis de primeira, segunda, e terceira ordem, de acordo com Gujarati e Porter (2011) é comum a existência de autocorrelação em séries temporais quando o intervalo de tempo da coleta de dados é curto, como no caso de dados coletados diariamente, semanalmente, mensalmente. Como não é desejável que um modelo apresente autocorrelação, e os dados apresentaram correlação de primeira ordem, foi necessário corrigi-lo utilizando o modelo de Cochrane-Orcutt.

\section{Apresentação da empresa}

O estudo de caso foi realizado em um Centro de Distribuição Direta (CDD) de bebidas. O CDD estudado pertence a uma multinacional, está localizado no sul da Bahia e é responsável pelo abastecimento de toda essa região.

As atividades principais realizadas pela unidade são a efetivação do pedido do cliente e a distribuição dos produtos para estes. Para tal, além da estrutura logística, a unidade apresenta ainda um setor de vendas e outros setores responsáveis pelo suporte aos serviços como Financeiro, Recursos Humanos e Marketing, contando atualmente com um total de 76 funcionários próprios.

A empresa em geral adota para os serviços de transporte a parceria com empresa terceirizada, responsável pelo fluxo das bebidas da fábrica ao CDD e pela entrega aos clientes. Essa terceirizada conta com um quadro de 150 funcionários e uma frota de 30 caminhões. Quando necessário, há ainda a contratação de pessoas autônomas que trabalham com movimentação de carga, sendo este tipo de serviço responsabilidade da transportadora.

O fluxo de bebidas da fábrica ao CDD é feito baseado na previsão de vendas para o mês vigente estabelecida até três meses antes, considerando a meta de volume de vendas estabelecida no inicio de cada ano. Em média, mensalmente, há a movimentação de cerca de 22.000 hectolitros de bebidas entre o recebido pela fábrica e entregue ao consumidor. Este é o principal resultado da empresa e a garantia de que os processos estão sendo cumpridos devidamente.

Para garantir o volume de vendas da operação é necessário que o vendedor visite e efetue a venda para o máximo de clientes de sua base, independente do seu tamanho e potencial. Além disso, é possível que o vendedor visite e efetue venda para clientes que não estejam na base do dia, o que 
deve acontecer em casos de a venda ser de fato importante para influenciar no resultado de volume. Para concluir o processo de venda deve-se garantir que o produto chegue ao cliente evitando a devolução da bebida ao CDD.

As operações de vendas e logística são as responsáveis pelo contato com o cliente. É a partir deste contato que o cliente desenvolve suas opiniões acerca dos serviços prestados pela empresa e estabelece sua satisfação com estes. Sendo assim, a qualidade de ambos os serviços é constantemente monitorada através de indicadores de desempenho. Os principais indicadores são classificados como indicadores de produtividade, ou seja, são parâmetros para medir a produção visando aferir o desempenho da empresa, e são eles: percentual de vendas efetuadas aos clientes da base, percentual de devolução e percentual de fora de rota, e visam o alcance de resultados operacionais satisfatórios, sendo o volume de vendas o principal. Todos eles serão apresentados a seguir.

\section{Apresentação das variáveis}

\subsection{Indicador de resultado: volume de vendas}

O volume de vendas é o indicador de desempenho mais importante da empresa, uma vez que é o responsável por determinar o faturamento. Portanto, é a partir da análise do crescimento do volume de vendas que se define se a companhia está tendo o desempenho operacional esperado ou se é necessário obter melhorias.

O volume de bebidas é medido em hectolitros, sendo que cada hectolitro equivale a 100 litros. Essa quantidade é analisada para cada tipo de embalagem que a empresa possui, por exemplo, no caso da venda de dez caixas com 24 garrafas de $600 \mathrm{~mL}$, a empresa possui um volume de 144 litros que corresponde a 1,44 hectolitros. É importante ressaltar que o foco não deve ser apenas alcançar a meta de volume total, mas também disponibilizar ao mercado todo o leque de produtos da empresa.

Para se obter um maior controle sobre esse indicador tão importante, a companhia estabelece uma meta de volume anual para todas as suas unidades. Essa meta anual é dividida para todos os meses, a depender das características de demanda de cada região. As metas de volume definidas são divididas para os vendedores no inicio do mês a depender da área e do histórico de compra dos pontos de vendas que atende.

A figura 1 apresenta os valores de volume em hectolitros no período de 30 meses ( 20132014-2015). É possível observar que os períodos com maior volume são os meses de dezembro e 
ISSN: $1415-7314$

ISSN online: $2317-6717$

janeiro, acompanhados pelo mês de junho. Isso porque o CDD está localizado em região litorânea, em que a demanda por bebidas é maior no período do verão. Além disso, o fato do CDD atender às cidades vizinhas em que acontecem as festividades juninas faz com que o volume em junho seja elevado. O mês em que há o menor volume de vendas é setembro, pois é caracterizado por uma considerável redução de frequência às praias e não há festividades que influencie o aumento das vendas.

Figura 1 - valores de volume de vendas mensal

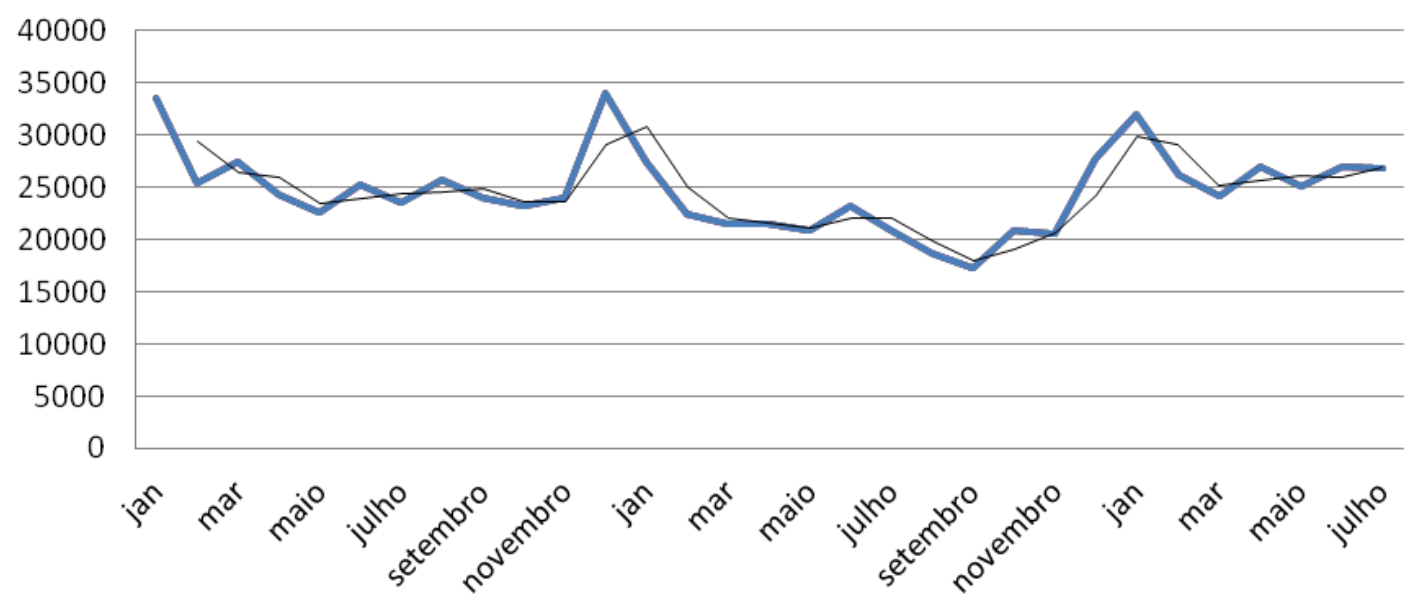

Além de fatores como clima da região ou época do ano, há diversos fatores que podem influenciar o crescimento ou redução deste indicador, e estes devem ser monitorados com muita atenção. Para o estudo atual, serão descritos três indicadores que podem influenciar o volume de vendas e que serão consideradas as variáveis de processo.

\section{Apresentação das variáveis de processo}

Os indicadores identificados como variáveis independentes e escolhidos para esse estudo estão relacionados com os principais processos da empresa e fazem parte de um grupo de indicadores classificados como indicadores de performance. Aqueles que fazem parte dessa classificação, porém, não foram selecionados, fazem menção a grupos específicos de produtos e embalagens da empresa, e possuem analise de volume individual. Uma vez que esse trabalho objetiva estudar a relação dos indicadores com o volume total de vendas, que considera o somatório do resultado de volume de todos os produtos optou-se por analisar indicadores que sejam genéricos, sendo eles: percentual de vendas efetuadas aos clientes da base, percentual de devolução e percentual de fora de rota.

\subsection{Percentual de vendas efetuadas aos clientes da base}

Devido a algumas situações que podem impossibilitar o contato direto com o cliente, como ausência deste no ponto de vendas, cliente sem disponibilidade para comprar, estoque cheio etc.

ENGEVISTA, V. 18, n. 1, p. 125-141, Julho 2016. 
estabeleceu-se que um número aceitável de clientes a efetuar compra de qualquer produto disponível pela empresa seja de $85 \%$ do número total de visitas programadas diariamente. Ainda que o cliente seja contatado por telefone, a efetuação da venda é válida para a análise do indicador em questão.

A figura 2 apresenta os valores desse indicador no período analisado.

Figura 2 - Percentual de vendas efetuadas aos clientes da base mensal

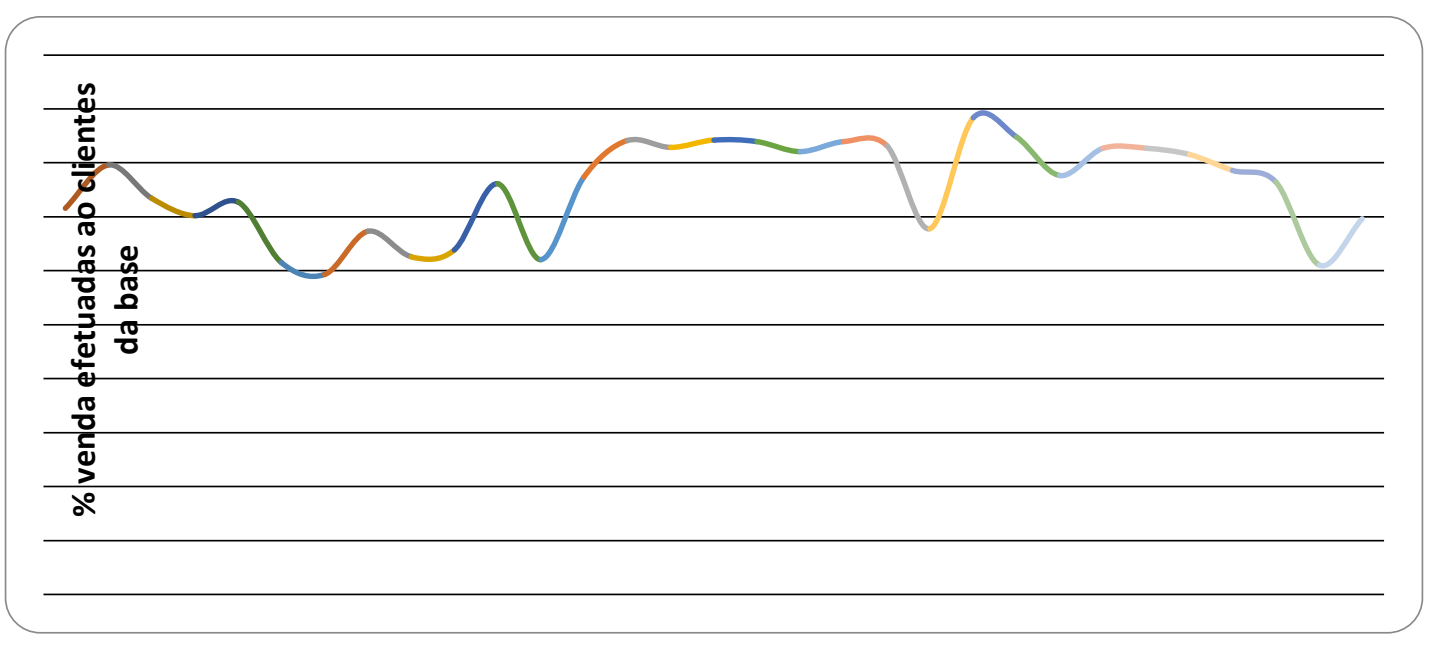

\subsection{Percentual de devolução}

O retorno para o armazém dos produtos que deveriam ser entregues ao cliente é indesejável. Muitas vezes, no momento da entrega, é possível que o cliente não esteja presente para receber, ou pode ter acontecido algum erro no momento da venda como condição de pagamento, diferença entre o produto solicitado pelo cliente e o produto que está sendo entregue, ou mesmo cliente não possuir o dinheiro para pagar no momento da entrega ou ainda a entrega chegar além do horário prometido e o cliente se recusar a receber. Todos esses casos provocam a devolução dos produtos, e a tolerância para tal é de 4,95\% de caixas devolvidas. É necessário que a venda do dia anterior seja efetuada corretamente e que todos os detalhes sejam alinhados previamente com o cliente que receberá o produto.

Para o cálculo do indicador, deve-se considerar diariamente a quantidade carregada em cada caminhão e o quanto daquela carga total retornará para o CDD. O cálculo então é feito a partir de:

$$
\% \text { de devolução }=\frac{\begin{array}{c}
\text { número total de caixas } \\
\text { que retornam ao CDD }
\end{array}}{\begin{array}{c}
\text { número total de caixas } \\
\text { carregadas nos caminhões }
\end{array}}
$$

O percentual de devolução mensal do período está representado na figura 3. 


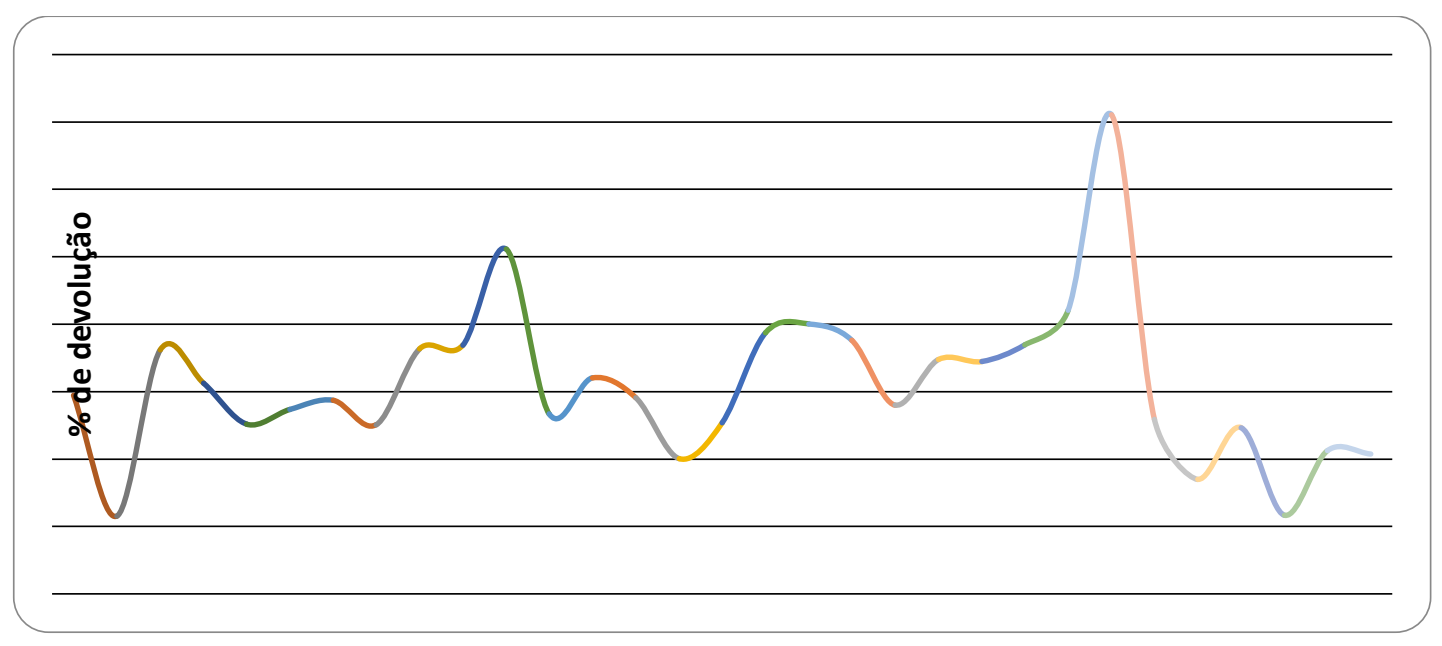

\subsection{Percentual de fora de rota}

Cada cliente possui um ou dois dias da semana de visita de acordo com sua disponibilidade de compra e com a proximidade entre os pontos de vendas que o vendedor atende. Estes devem ser atendidos apenas em seu dia de visita. Porém, em alguns casos, eles solicitam atendimentos em dias diferentes pelo fato de não estarem disponíveis em seu dia de visita habitual, ou por haver algum tipo de promoção no dia diferente daquele que recebe a visita. Esses casos extras são considerados como fora de rota, com uma meta máxima de $15 \%$ que não deve ser ultrapassada, uma vez que influencia diretamente nos custos logísticos já que impõem um desvio na rota programada.

Na Figura 4 estão contidos os valores do período analisado para esse indicador.

Figura 4 - valores de percentual de fora de rota mensal

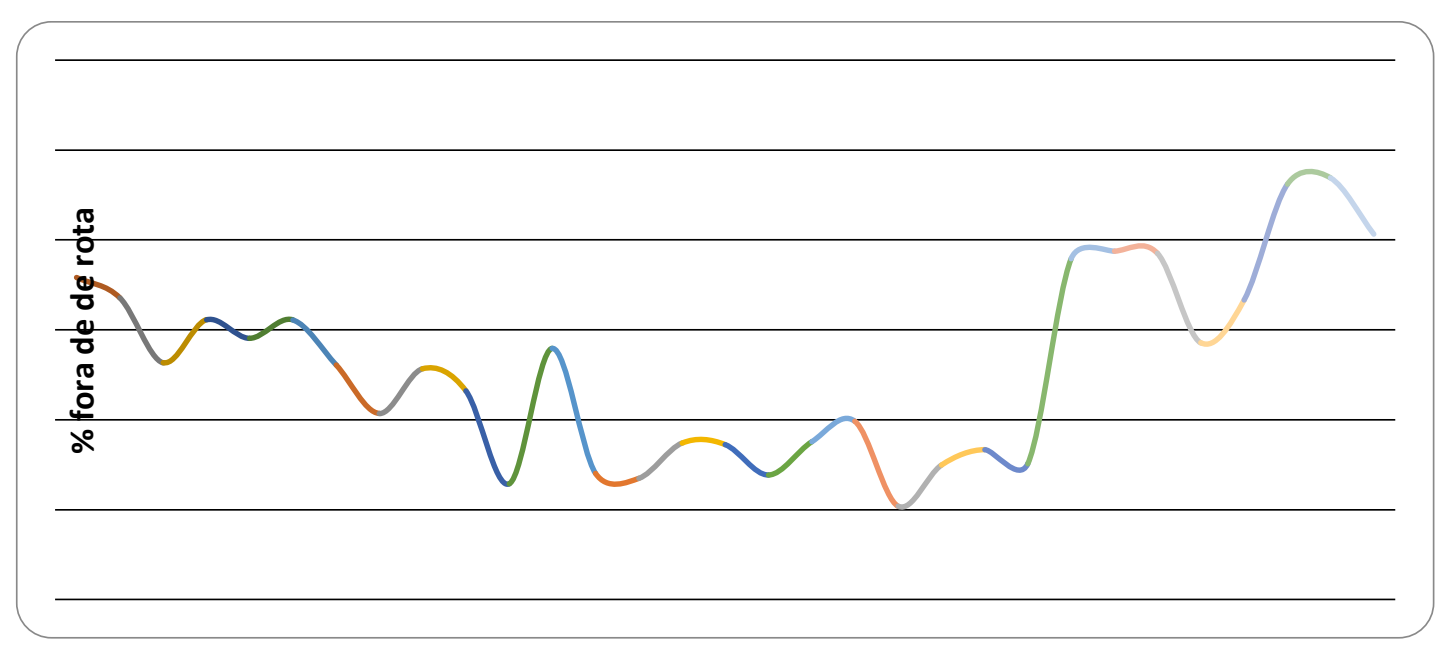

\section{Descrição dos resultados}

Para o tratamento do conjunto de todas as variáveis será usada a seguinte nomenclatura: o $\mathrm{Y}$ representa a váriavel dependente volume de vendas, X1 variavel independente \% de entregas fora de rota, X2 \% devolução, e X3 \% de vendas efetuadas direto ao cliente. 
A tabela 1 resume o resultado do modelo dos mínimos quadrados, verifica-se que X1 e X2 são significativas, sendo que X1 é significativa ao nível de 1\%, e X2 ao nível de 5\%, enquanto que X3 não foi significativa. Entretanto de acordo com o modelo, se excluirmos a constante aumenta-se a significância de X3.

O valor de r2 foi de 0,400558 o que significa que apenas $40 \%$ das variações de y estão sendo explicadas pelos coeficientes de $\mathrm{X}$.

Analisando a estatística-F $(3,27)=7,68218(\mathrm{p}$-valor $=0,000724)$, podemos rejeitar a hipótese nula, que diz que nenhuma das variáveis analisadas afetam o volume de vendas, como essa hipótese esta sendo rejeitada, conclui-se que ao menos uma das variáveis estão afetando o volume de vendas.

Tabela 1: Modelo dos mínimos quadrados com constante

\begin{tabular}{|l|}
\hline Hipótese nula: a especificação é adequada \\
\hline Estatística de teste: $\mathrm{F}(2,25)=0,0331306$ \\
\hline $\mathrm{p}$-valor $=\mathrm{P}(\mathrm{F}(2,25)>0,0331306)=0,967455$ \\
\hline
\end{tabular}

Utilizando o mesmo modelo, sem a constante, o resultado do modelo melhora significativamente, todas as variáveis passam a ser significativas, sendo que X1 e X3 ao nível de 1\%, e X2 ao nível de 5\%, analisando Estatística-F $(3,28)=508,332$ (p-valor < 0,00001), rejeita-se novamente a hipótese nula, e conclui-se que ao menos uma das variáveis afetam o volume de vendas. E o valor de $\mathrm{r} 2$ quanto de $\mathrm{r} 2$ ajustado passa a ser de 0,98 .

Através do resultado do teste de RESET ( regression especificacion error test), verifica-se que o modelo é adequado, com o p-valor de 0,967455, ou seja, não há erros de especificação presentes no modelo, tais como omissão de variável relevante, inclusão de variável irrelevante, ou mesmo erros de medida.

Ao analisarmos o resultado do teste de White, que encontra-se resumido na tabela 2, verificamos que a hipótese nula é aceita, ou seja, não há heterosquedicidade, temos homocedasticia como desejado, com $\mathrm{p}$ valor de 0,579 .

Tabela 2: Resumo do teste de White

\begin{tabular}{|l|}
\hline Teste de White para a heterosquedicidade - \\
\hline Hipótese nula: sem heterosquedicidade \\
\hline Estatística de teste: $\mathrm{TR}^{\wedge} 2=7,55813 \mathrm{com}$ - \\
valor $=\mathrm{P}($ Chi-Square $(9)>7,55813)=$ \\
0,579214 \\
\hline
\end{tabular}

Em relação à existência de autocorrelação entre as variáveis, é possível verificar que existe autocorrelação quando a defasagem é de 1 período, rejeitando-se a hipótese nula. Já para segunda ordem não há autocorrelação, assim como para terceira ordem também não, o resultado dos testes estão resumidos na tabela 3 . 
Tabela 3: Resumo dos teste de autocorrelação

\begin{tabular}{|c|}
\hline \\
\hline Hipó \\
\hline $\begin{array}{l}\text { Estatística de teste: } \mathrm{LMF}=6,27023 \text { com } p \text {-valor } \\
=\mathrm{P}(\mathrm{F}(1,25)>6,27023)=0,0191683\end{array}$ \\
\hline Teste LM para autocorrelação até à ordem 2 \\
\hline Hipót \\
\hline $\begin{array}{l}\text { Estatística de teste: } \mathrm{LMF}=2,88099 \text { com } \mathrm{p} \text {-valor } \\
=\mathrm{P}(\mathrm{F}(2,23)>2,88099)=0,0764635\end{array}$ \\
\hline Teste LM para autocorrelação até à ordem 3 \\
\hline Hipótese nula: sem autocorrelação \\
\hline $\begin{array}{l}\text { Estatística de teste: } \mathrm{LMF}=2,06058 \text { com } \mathrm{p} \text {-valor } \\
=\mathrm{P}(\mathrm{F}(3,21)>2,06058)=0,136135\end{array}$ \\
\hline
\end{tabular}

Como o ideal para o modelo é que não exista autocorrelação, é necessário corrigir a correlação encontrada na primeira ordem, para isso foi utilizado um segundo modelo de CochraneOrcutt, e obteve-se o resultado apresentado na tabela 4. O coeficiente de correlação de primeira ordem passou a ser de 0,06, o que significa que ao nível de significância de 95\% não existe correlação. Entretanto, assim como aconteceu no modelo dos mínimos quadrados com a presença da constante a variável X3 não foi significativa, e o valor de r2 ajustado foi de 0,51, após a exclusão da constante o p-valor de X3 aumentou e todas as variáveis foram significativas ao nível de $1 \%$. No entanto ao excluir a constante do modelo o problema da correlação de primeira ordem entre as variáveis reapareceu, e o r2 ajustado diminuiu para 0,43 , por essas duas razões foi testado o mesmo modelo excluindo X3 ( conforme pode ser observado na tabela 5), e mantendo-se a constante, nesse caso não apareceu a correlação de primeira ordem, e o r2 ajustado foi de 0,51.

Tabela 4: Modelo de Cochrane Orcutt com constante

\begin{tabular}{|lcccc|}
\hline Variável dependente: Y & & & \\
\hline $\begin{array}{l}\text { VARIÁVEL } \\
\text { VALOR }\end{array}$ & COEFICIENTE & ERRO PADRÃO & ESTAT. T & P- \\
\hline const & 19375,0 & 6183,89 & 3,133 & $0,00425 * * *$ \\
\hline X1 & 44332,4 & 12809,5 & 3,461 & 0,00187 *** \\
\hline X2 & 48552,4 & 20914,1 & 2,322 & $0,02836 * *$ \\
\hline X3 & $-5122,86$ & 6747,39 & $-0,759$ & 0,45454 \\
\hline
\end{tabular}

Tabela 5: Modelo de Cochrane Orcut excluindo a variável X3

\begin{tabular}{|ccccc|}
\hline $\begin{array}{l}\text { VARIÁVEL } \\
\text { VALOR }\end{array}$ & COEFICIENTE & ERRO PADRÃO & ESTAT. T & P- \\
\hline $\begin{array}{c}\text { const } \\
* * *\end{array}$ & 14990,1 & 2295,03 & 6,532 & $<0,00001$ \\
\hline $\begin{array}{c}\text { X1 } \\
* * *\end{array}$ & 48259,0 & 11748,1 & 4,108 & 0,00033 \\
\hline $\begin{array}{c}\text { X2 } \\
* *\end{array}$ & 48766,2 & 20716,9 & 2,354 & 0,02611 \\
\hline
\end{tabular}


No entanto ao excluir a constante do modelo o problema da correlação de primeira ordem entre as variáveis reapareceu, e o r2 ajustado diminuiu para 0,43 , por essas duas razões foi testado o mesmo modelo excluindo X3 ( conforme pode ser observado na tabela 5), e mantendo-se a constante, nesse caso não apareceu a correlação de primeira ordem, e o r2 ajustado foi de 0,51 .

Também foi realizado o teste de multicolinearidade cujo o objetivo é que o fator de inflação da variância seja o mais baixo possível, e para todas as variáveis o valor foi próximo a 1 , indicando que existe pouca ou nenhuma colinearidade entre as variáveis, conforme desejado: X1 $=1,174 ; \mathrm{X} 2=1,065 ; \mathrm{X} 3=1,132$.

Diante dos resultados o modelo escolhido foi o de Chrocane - Orcut excluindo-se X3, dessa maneira, com a aceitação desse modelo, percebe-se que as entregas fora de rota ocasionam um aumento de 48259 hectolitros, a variável devolução ocasiona um incremento no volume de vendas de 48766 sendo que esse modelo explica 51\% do volume de vendas.

\section{Considerações finais}

As análises realizadas foram essenciais para determinar a validade da hipótese levantada no início da pesquisa, ou seja, que os indicadores que a empresa utiliza refletem no volume de vendas.

Os resultados obtidos a partir das análises foram condizentes com o esperado, e o modelo escolhido explica $51 \%$ do volume de vendas, entretanto X3 não teve significância estatística. Considerando que os indicadores possuem a função de traduzir os procedimentos operacionais em resultados para a empresa, deve-se considerar a possibilidade de revisão desse indicador e realizar análise mais profunda acerca da validade e credibilidade da informação gerada pelos indicadores adotados pela empresa.

Vale ressaltar que a empresa adota o r outros indicadores, específicos a cada linha de produto, é possível que haja relação entre esses e os indicadores de produtividade aqui estudados. Assim, os parâmetros que não foram aqui expostos podem ser responsáveis indiretamente pela variação do resultado de volume de vendas.

Os indicadores de produtividade podem ainda ser relacionados com outros indicadores de resultados como é o caso do indicador de market share, que demonstra a participação dos produtos da empresa no mercado comercial. É possível que haja relação maior entre eles, o que sugere a possibilidade de realização de pesquisa para avaliar tal hipótese.

Além disso, há outros indicadores que podem ser analisados para comprovar a influência ou não nos resultados de volume de vendas como: compra mensal dos pontos de vendas de grande porte, eventos mensais nos pontos de vendas, utilização de equipamentos de refrigeração, etc. Todos estes estão de alguma forma relacionados ao aumento da comercialização dos produtos da empresa e podem ajudar a explicar o comportamento do volume. 
É possível concluir que para estudar o comportamento de processos e resultados em uma empresa faz-se necessário considerar diversas variáveis que podem influenciá-los, nesse aspecto o uso de ferramentas estatísticas adequadas podem auxiliar na escolha do melhor sistema de medição de desempenho. Para isso, a empresa deve estar comprometida a desenvolver e implementar indicadores que revelem seu cenário real e que contribuam para a gestão do seu desempenho e conseqüente aprimoramento.

\section{Referências}

CORREA, S.M.B.B.; Probabilidade e estatística. $2^{\mathrm{a}}$ edição. Belo Horizonte. PUC Minas Virtual. 2003. 116 p.

CORTÊS, A. F.; Sistema de indicadores de desempenho logístico de um centro de distribuição do setor supermercadista. Dissertação. Universidade Federal de Santa Catarina. 2006.

FRANCISCHINI, P. G.; MIYAKE, D. I. \& GIANNINI, R.; Adaptação de conceitos de melhorias operacionais provenientes do Lean Production em operações de serviços. Anais... XXVI Encontro Nacional de Engenharia de Produção - ENEGEP. Fortaleza CE. Brasil. Outubro de 2006.

FREITAS, A. L. P.; A qualidade em serviços no contexto da competitividade. Produção Online. v. 5 n. 1.2005.

FREITAS, A. KPI - Indicador de desempenho de Processo. Disponível em: <www.academiaplatonica.com.br>. Acesso em: 16 de novembro de 2014.

GONÇALVES, J. P. Desempenho Organizacional. Seminário Econômico. São Paulo. n. 815. 2002.

GUIMARÃES, P. R. B.; Métodos Quantitativos Estatísticos. Curitiba. IESDE Brasil S.A. 2008. 245p.

KAPLAN, R. S.; NORTON, D. P.; Using the Balanced Scorecard as a Strategic Management System. Harvard Business Review. 2007.

KAPLAN, R.S. \& NORTON, D. P.; Strategy Maps: Converting Intangible Assets into Tangible Outcomes. Harvard Business Press Books. 2004.

MARTINS, R. A. \& COSTA NETO, P. L. O.; Indicadores de desempenho para a gestão pela qualidade total: uma proposta de sistematização. Gestão da Produção. v5. n3. P 298311. 1998.

MASCULO, F. S.; Um panorama da engenharia de produção. Associação Brasileira de Engenharia de Produção - ABEPRO. 2006. Disponível em: <www.abepro.org.br>. Acesso em: 10 de outubro de 2014.

MONTGOMERY, D. C.; Introdução ao controle estatístico da qualidade. Rio de Janeiro: LTC, 2004

OLIVEIRA, F. E. M.; Estatística e probabilidade. $2^{\mathrm{a}}$ edição. Editora Atlas. 2007.

PARASURAMAN, A.; ZEITHAML, V. A. \& BERRY, L. L. SERVQUAL: Multipleitem scale for measuring consumer perceptions of service quality. Journal of Retailing, v. 64, n. 1, p. 12-40, 1988.

PARMENTER, D.; Key Performance Indicators: developing, implementing, and using winning KPIs. Hoboken, New Jersey: John Wiley \& Sons, 2010. 
ROEST, P.; The golden rules for implementing the Balanced Business Scorecard. Information Management \& Computer Security. MCB University Press. 1997.

SANTOS, A. Centros de distribuição como vantagem Competitiva. Revista de Ciencias Gerenciais. Anhanguera Educacional. SARE- Sistema Anhaguera de Revistas Eletrônicas. V 10. N 12. 2006.

SILVA, B.B.; DUARTE, E. \& MANO, A. P.; Utilização de ferramentas de melhoria continua para realizar a gestão de uma disciplina de um curso de engenharia de produção. VIII Congresso Nacional de Excelência em Gestão. 2012.

SLACK, N.; CHAMBERS, S.; HARLAND, C.; HARRISON, A. \& JOHNSTON, R.; Administração da Produção. São Paulo. Atlas. 1997.

SOARES, T. D. L. V. A. M. \& RATTON, C. A.; Medição de desempenho e estratégias orientadas para o cliente: resultados de uma pesquisa de empresas líderes no Brasil. RAE - Revista de Administração de Empresas. v39. n.4. 1999.

SOUZA, G. V.; Mudanças organizacionais de bens para serviços. Monografia. Universidade Veiga De Almeida. Gabriel Viana De Souza. 2006.

STEVENSON, W. J.; Estatística aplicada à Administração. Harper \& Row do Brasil, São Paulo, 1981.

TEZZA, R.; BORNIA, A.C. \& VEY, I.H.; Sistemas de medição de desempenho: uma revisão e classificação da literatura. Gestão da Produção, São Carlos, v. 17, n. 1, p. 7593, 2010. 